Pacific Journal of Mathematics

HOMOGENEOUS SPECTRAL SETS AND LOCAL-GLOBAI 


\title{
HOMOGENEOUS SPECTRAL SETS AND LOCAL-GLOBAL METHODS IN BANACH ALGEBRAS
}

\author{
ANGEL LAROTONDA AND IGNACIO ZALDUENDO
}

The search for topological characterization of algebraic properties of commutative Banach algebras has often led to the calculation of cohomology groups of its spectrum.

In the present paper, a tool is developed which permits the application of sheaf cohomological methods to such problems by lifting sequences of complex analytic manifolds to sequences of spectral sets and cohomology groups.

Introduction. Suppose $A$ is a commutative, unital Banach algebra, with spectrum $X(A)$, and let

$$
\theta: \mathscr{O}(X(A)) \rightarrow A
$$

be the global functional calculus defined by Craw [3]. Also, let $M$ denote a complex analytic submanifold of $\mathbf{C}^{n}$.

While searching for characterizations of Čech cohomology groups of $X(A)$, Novodvorskii [8] presented the spectral sets

$$
A^{M}=\left\{a=\left(a_{1}, \ldots, a_{n}\right) \in A^{n}: \operatorname{sp}\left(a_{1}, \ldots, a_{n}\right) \subset M\right\} .
$$

Later Taylor used in [10]

$$
A_{M}=\left\{a=\left(a_{1}, \ldots, a_{n}\right) \in A^{n}: a=\theta^{n}(f) \text {, with } f \in \mathscr{O}(X(A), M)\right\} .
$$

As it turns out, such sets have a very rich structure. It was proved in [6] that they are Banach manifolds modelled on the projective $A$-modules of $\operatorname{rank}=\operatorname{dim}(M)$.

Our aim in this note is to extend the definition of spectral set to include manifolds such as the complex projective line, or complex Grassmann manifolds, which are not submanifolds of $\mathbf{C}^{n}$. We will obtain, for example, a good definition of meromorphic elements of a Banach algebra. By "good" we mean that our definition will be intrinsic, will coincide with Taylor's definition for submanifolds of $\mathbf{C}^{n}$, and will verify a Novodvorskii-Taylor type theorem [10] when the manifold is a homogeneous space. We shall also generalize Craw's global function calculus $\theta$ to a map

$$
\theta_{M}: \mathscr{O}(X(A), M) \rightarrow A_{M}
$$


Here $\mathscr{O}(X(A), M)$ is the set of germs of analytic functions near $X(A)$ with values in $M$, as defined in [7].

The problem has led us to the use of sheaves of "local" spectral sets and sheaf cohomology. Section 1 is a very brief presentation of the facts we shall need about analytic functions over the dual space of $A$, and their sheaves. In $\S 2$ the localization of Banach algebras which we shall use is developed. In $\S \S 3$ and 4 the local spectral sets and their sheaf over $X(A)$ are defined. The intrinsic definition of $A_{M}$ for any complex analytic manifold $M$ (Definition 5.1) is given in $\S 5$. It is proved that this definition coincides with Taylor's when $M$ is a submanifold of $\mathbf{C}^{n}$. A Novodvorskii-Taylor theorem (Theorem 5.9) is obtained, as well as generalized Gelfand transform, spectrum, and functional calculus. In $\S 6$ we take a closer look at spectral sets constructed from Lie groups and homogeneous spaces, and our main theorem (Theorem 6.3) regarding the inexactness of "lifted" exact sequences is obtained. Finally, a few consequences are presented in $\S 7$.

1. Analytic functions over the dual space. Let $U$ be an open subset, in the weak *-topology, of the dual space $A^{\prime}$ of $A$. We shall say a function

$$
f: U \rightarrow \mathbf{C}
$$

is analytic if it is locally bounded and all its directional derivatives exist throughout $U$ [1]. The complex algebra of all such maps will be denoted by $\mathscr{O}(U)$. For example the linear maps $\tilde{a}: A^{\prime} \rightarrow \mathbf{C}$ given by $\tilde{a}(\gamma)=\gamma(a)$ belong to $\mathscr{O}\left(A^{\prime}\right)$ for all $a$ in $A$.

Consider, for $\gamma \in A^{\prime}$, the set of germs of $\mathbf{C}$-valued analytic maps on $\gamma$. This is a local algebra which we will denote by $\mathscr{O}_{\gamma}$. The local boundedness of analytic functions makes it possible to consider each germ as depending only on a finite number of variables [1]; $\mathscr{O}_{\gamma}$ may be presented in consequence as a direct limit of algebras of germs of $\mathrm{C}$-valued analytic maps defined on finite dimensional spaces. More precisely: if $V$ is a finite dimensional subspace of $A$, the transpose

$$
j: A^{\prime} \rightarrow V^{\prime}
$$

of the inclusion map, induces a mapping $f \mapsto f \circ j$ between the spaces of analytic functions.

If $\mathscr{O}$ and $\mathscr{O}_{V}$ are the sheaves of analytic functions on $A^{\prime}$ and $V^{\prime}$ respectively, we have a subsheaf $V_{V} \mathscr{O}=j^{*}\left(\mathscr{O}_{V}\right)$ of $\mathscr{O}$. Clearly, if $f \in H^{0}(U, \mathscr{O})$, then $f \in H^{0}\left(U,{ }_{V} \mathscr{O}\right)$ if and only if it can be locally factored as $g \circ j$ for some analytic functions $g$ defined on open subsets of $V^{\prime}$. Hence, we have $\mathscr{O}=\underline{\lim }_{V} \mathscr{O}$, where $V$ ranges over all finite 
dimensional subspaces of $A$. Also, if $K$ is any compact set in $A^{\prime}$, we have $H^{0}(K, \mathscr{O})=\lim _{\longrightarrow} H^{0}(K, V \mathscr{O})$; and if we denote $K_{V}=j(K)$ we have an obvious isomorphism

$$
H^{0}\left(K_{V}, \mathscr{O}_{V}\right) \rightarrow H^{0}(K, V \mathscr{O})
$$

induced by the map $j[\mathbf{1 1}]$.

Let $P\left(V^{\prime}\right)$ denote the algebra of functions on $V^{\prime}$ generated by the linear maps $\tilde{a}, a \in V$. Then $P\left(A^{\prime}\right)=\underline{\lim } P\left(V^{\prime}\right)$ in a natural way, where $V$ ranges over all finite dimensional subspaces of $A$.

The canonical map $a \mapsto \tilde{a}$ shows that $P\left(V^{\prime}\right)$ is isomorphic to the symmetric algebra of the vector space $V$. The algebra morphisms

$$
P\left(V^{\prime}\right) \rightarrow A
$$

induced by the inclusions are compatible, producing an algebra morphism

$$
P\left(A^{\prime}\right) \rightarrow A \text {. }
$$

This morphism extends uniquely to a surjective morphism

$$
\theta: \mathscr{O}(X(A)) \rightarrow A
$$

and this map is the functional calculus [9].

2. The sheaf $\mathscr{A}$. Let $\Omega$ be a polynomially convex open subset of $V^{\prime}$, and $U=j^{-1}(\Omega)$. Open subsets of $A^{\prime}$ such as this one are called $A$ convex and finitely determined. The spectrum $X(A)$ of $A$ has a basis for neighborhoods made up of such sets [7].

Suppose $U$ is any $A$-convex finitely determined neighborhood of $X(A)$, and let $I_{V, U}$ denote the kernel of the functional calculus map

$$
\mathscr{O}(j(U)) \rightarrow A \text {. }
$$

If $\mathscr{J}_{V, U}$ is the corresponding subsheaf of $\mathscr{O}_{V}$, we have

$$
\begin{aligned}
& H^{0}\left(j(U), \mathscr{J}_{V, U}\right)=I_{V, U} \text { and } \\
& H^{r}\left(j(U), \mathscr{J}_{V, U}\right)=0, \quad \text { for all } r \geq 1 .
\end{aligned}
$$

Now let $\mathscr{J}_{U}$ be the subsheaf $\lim j^{*}\left(\mathscr{J}_{V, U}\right)$ of $\mathscr{O}$. Then

$$
\begin{aligned}
& H^{0}\left(U, \mathscr{J}_{U}\right)=I_{U}, \text { the kernel of } \mathscr{O}(U) \rightarrow A, \quad \text { and } \\
& H^{r}\left(U, \mathscr{J}_{U}\right)=0, \quad \text { for all } r \geq 1 .
\end{aligned}
$$

Finally, let $\mathscr{N}=\lim _{\longrightarrow} \mathscr{J}_{U}$, where $U$ ranges over all $A$-convex finitely determined open neighborhoods of $X(A), \mathscr{N}$ is the subsheaf of $\mathscr{O}$ whose stalk $\mathscr{N}_{\gamma}$ over $\gamma$ is the ideal of $\mathscr{O}_{\gamma}$ generated by elements of 
$N=\operatorname{Ker} \theta$. Note that if $f$ belongs to $\mathscr{N}_{\gamma}$ then $f$ also belongs to $\mathscr{N}_{\psi}$, for all $\psi$ sufficiently close to $\gamma$. Note also that

$$
\begin{aligned}
& H^{0}(X(A), \mathscr{N})=N \text { and } \\
& H^{r}(X(A), \mathscr{N})=0, \text { for all } r \geq 1 .
\end{aligned}
$$

Now we are interested in the sheaf $\mathscr{A}=\mathscr{O} / \mathscr{N}$. We have the following proposition.

2.1. Proposition. (i) The stalk $A_{\gamma}$ of $\mathscr{A}$ over $\gamma$ is zero if $\gamma \notin X(A)$. Therefore, we may consider $\mathscr{A}$ as a sheaf over $X(A)$.

(ii) $H^{0}(X(A), \mathscr{A})=A$, and $H^{r}(X(A), \mathscr{A})=0$ for all $r \geq 1$.

Proof. Suppose $\gamma \notin X(A)$. There exists some $a \in A$ such that the map

$$
u: A^{\prime} \rightarrow \mathbf{C}, \quad \text { defined by } u(\psi)=\psi(a)^{2}-\psi\left(a^{2}\right)
$$

verifies $u(\gamma)=1$. But clearly $\theta(u)=0$, so the germ of $u$ at the point $\gamma$ belongs to the ideal $\mathscr{N}_{\gamma}$ of $\mathscr{O}_{\gamma}$. Since this germ is invertible in $\mathscr{O}_{\gamma}$, it follows that $\mathscr{N}_{\gamma}=\mathscr{O}_{\gamma}$, and $A_{\gamma}=0$.

The second statement follows from the exact cohomology sequence associated to the sheaf sequence

$$
0 \rightarrow \mathscr{N} \rightarrow \mathscr{O} \rightarrow \mathscr{A} \rightarrow 0
$$

considering that $H^{r}(X(A), \mathscr{O})=0$, for all $r \geq 1$, and the cohomology of $\mathscr{N}$ referred to above.

If $\mathscr{C}$ denotes the sheaf of continuous $\mathrm{C}$-valued functions over $X(A)$, there is a map

$$
\left.\mathscr{A}\right|_{X(A)} \rightarrow \mathscr{C}
$$

which may be considered a sheaf form of the Gelfand transform.

3. The local spectral sets. We will now define the local spectral sets $A_{M}(\psi)$.

Let $M$ be any complex analytic manifold, and $\psi \in X(A)$. Let

$$
S_{\psi}(M)=\left\{(m, \lambda): m \in M, \lambda: \mathscr{O}_{m} \rightarrow A_{\psi}\right\} .
$$

Here $\mathscr{O}_{m}$ is the algebra of germs of complex valued analytic functions defined near $m$, and $\lambda$ is an algebra morphism. Let $\mathscr{O}_{\psi}^{M}$ denote the set of $M$-valued analytic germs near $\psi$, and put

$$
\alpha_{\psi}: \mathscr{O}_{\psi}^{M} \rightarrow S_{\psi}(M) / \alpha_{\psi}(f)=\left(f(\psi), \theta_{\psi} \circ f^{*}\right)
$$

where $f^{*}(h)=h \circ f$, and $\theta_{\psi}: \mathscr{O}_{\psi} \rightarrow A_{\psi}$ is the canonical map. 
3.1. Definition. Let $\psi \in X(A)$, and $M$ be a complex manifold. If $\alpha_{\psi}$ is as above, $A_{M}(\psi)$ is the image of $\alpha_{\psi}$.

3.2. Proposition. Let $M$ and $N$ be complex analytic manifolds, and $h: M \rightarrow N$ an analytic map. Then $h$ induces a map

$$
h_{\psi}: A_{M}(\psi) \rightarrow A_{N}(\psi) .
$$

Moreover, if $d: N \rightarrow P$ is also analytic, $(d \circ h)_{\psi}=d_{\psi} \circ h_{\psi}$, and if id: $M \rightarrow M$ is the identity then $\mathrm{id}_{\psi}$ is the identity of $A_{M}(\psi)$.

Proof. To fix notation, let's say $A_{M}(\psi)$ is the image of $\alpha_{\psi}$ and $A_{N}(\psi)$ is the image of $\beta_{\psi}$. Let

$$
h_{\psi}\left(\alpha_{\psi}(f)\right)=\beta_{\psi}(h \circ f) .
$$

This is well defined, for if $\alpha_{\psi}(f)=\alpha_{\psi}(g), f(\psi)=g(\psi)$ and $\theta_{\psi} \circ f^{*}=$ $\theta_{\psi} \circ g^{*}$, hence $(h \circ f)(\psi)=(h \circ g)(\psi)$, and

$$
\theta_{\psi} \circ(h \circ f)^{*}=\theta_{\psi} \circ f^{*} \circ h^{*}=\theta_{\psi} \circ g^{*} \circ h^{*}=\theta_{\psi} \circ(h \circ g)^{*} \text {. }
$$

$(d \circ h)_{\psi}=d_{\psi} \circ h_{\psi}$ and id $\mathrm{id}_{\psi}=$ identity of $A_{M}(\psi)$ are easy consequences of the definition.

There is another, equivalent, definition for $A_{M}(\psi)$ : suppose $\operatorname{dim} M$ $=k$, and let $\mathscr{M}$ be a maximal atlas for $M$. A typical chart on $M$ will be denoted by $(x, \operatorname{dom} x)$, where $\operatorname{dom} x$ is the domain of $x$. Now define

$$
a_{\psi}: \mathscr{O}_{\psi}^{M} \rightarrow \prod_{\mathscr{M}}\left(A_{\psi}^{k} \cup\{\infty\}\right)
$$

by setting

$$
a_{\psi}(f)_{x}= \begin{cases}\theta_{\psi}^{k}(x \circ f), & \text { if } f(\psi) \in \operatorname{dom} x \\ \infty, & \text { if } f(\psi) \notin \operatorname{dom} x\end{cases}
$$

and define $A_{M}(\psi)$ as the image of $a_{\psi}$.

The equivalence of the two definitions may be seen by looking at the diagram

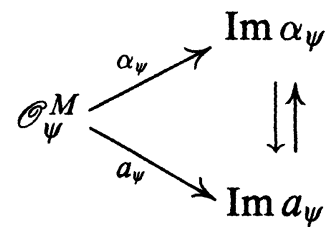

and the following lemma. 


\subsection{LEMMA. $a_{\psi}(f)=a_{\psi}(g)$ if and only if $\alpha_{\psi}(f)=\alpha_{\psi}(g)$.}

Proof. Suppose $a_{\psi}(f)=a_{\psi}(g)$. Knowing which coordinates of $a_{\psi}(f)$ are $\infty$ determines the point $f(\psi)$ (the only point of $M$ not belonging to the domains of these coordinates), so we have $f(\psi)=$ $g(\psi)$. Also, given any chart $x=\left(x_{1}, \ldots, x_{k}\right)$ near $f(\psi)$, the elements $x_{1}, \ldots, x_{k}$ generate the algebra $\mathscr{O}_{f(\psi)}$ topologically, and we have

$$
\begin{aligned}
& \left(\left(\theta_{\psi} \circ f^{*}\right)\left(x_{1}\right), \ldots,\left(\theta_{\psi} \circ f^{*}\right)\left(x_{k}\right)\right)=\theta_{\psi}^{k}(x \circ f) \\
& \quad=\theta_{\psi}^{k}(x \circ g)=\left(\left(\theta_{\psi} \circ g^{*}\right)\left(x_{1}\right), \ldots,\left(\theta_{\psi} \circ g^{*}\right)\left(x_{k}\right)\right)
\end{aligned}
$$

so $\theta_{\psi} \circ f^{*}=\theta_{\psi} \circ g^{*}$.

Now suppose $\alpha_{\psi}(f)=\alpha_{\psi}(g)$. The point $f(\psi)=g(\psi)$ determines all "infinite" coordinates. For all others we have

$$
\begin{aligned}
\theta_{\psi}^{k}(x \circ f) & =\left(\left(\theta_{\psi} \circ f^{*}\right)\left(x_{1}\right), \ldots,\left(\theta_{\psi} \circ f^{*}\right)\left(x_{k}\right)\right) \\
& =\left(\left(\theta_{\psi} \circ g^{*}\right)\left(x_{1}\right), \ldots,\left(\theta_{\psi} \circ g^{*}\right)\left(x_{k}\right)\right)=\theta_{\psi}^{k}(x \circ g)
\end{aligned}
$$

so $a_{\psi}(f)=a_{\psi}(g)$.

The manifold $M$ is contained in $A_{M}(\psi)$ as the image of the constant maps, and the projection onto the $x$-coordinate (which goes to $\mathbf{C}^{k} \cup$ $\{\infty\})$ may be identified with the chart $x$ itself. Thus $A_{M}(\psi)$ may be thought of as an extension of the manifold $M$. The projection onto the $x$-coordinate may be considered a "chart" on $A_{M}(\psi)$, which then has a manifold-like structure with tangent spaces modelled on $A_{\psi}^{k}$. We shall not need this structure on $A_{M}(\psi)$, and so will pursue it no further. We choose to work with $\operatorname{Im} \alpha_{\psi}$ to avoid the need for maximal atlases in specific examples of manifolds.

4. The sheaf $\mathscr{A}_{M}$ over the spectrum. We shall now define a sheaf of "manifolds" over the spectrum $X(A)$ of $A$. As the total space, put

$$
\mathscr{A}_{M}=\bigcup_{X(A)} A_{M}(\psi)
$$

and as the projection

$$
\pi: \mathscr{A}_{M} \rightarrow X(A), \quad \text { given by } \pi(u)=\psi \text { if } u \in A_{M}(\psi) .
$$

We will now define a topology on $\mathscr{A}_{M}$. Let $u \in \mathscr{A}_{M}$, and $\pi(u)=\psi$. There is an $f \in \mathscr{O}_{\psi}^{M}$ such that $u=\alpha_{\psi}(f)$. Suppose $f$ is defined over 
an open neighborhood $W$ of $\psi$, and let $s_{f}$ be the section of $\pi$

$$
s_{f}: W \rightarrow \mathscr{A}_{M}, \quad s_{f}(\psi)=\alpha_{\psi}(f) .
$$

We want $s_{f}(W)$ to be a neighborhood of $u$.

4.1. Proposition. $\left\{s_{f}(W): W\right.$ is open and $\left.f \in \mathscr{O}(W, M)\right\}$ is a basis for a topology on $\mathscr{A}_{M}$.

Proof. We must prove the following: if $u \in s_{f}(W) \cap s_{g}(V)$, and $\pi(u)=\psi$, then there is an $h \in \mathscr{O}_{\psi}^{M}$ and an open neighborhood $U$ of $\psi$ such that

$$
u \in s_{h}(U) \subset s_{f}(W) \cap s_{g}(V) .
$$

We have $s_{f}(\psi)=s_{g}(\psi)$, so $\alpha_{\psi}(f)=\alpha_{\psi}(g)$. We shall prove that this is a local property, that is, $\alpha_{\psi}(f)=\alpha_{\psi}(g)$ implies $\alpha_{\gamma}(f)=\alpha_{\gamma}(g)$ for all $\gamma \in X(A)$ which are sufficiently close to $\psi$.

Consider a chart $x=\left(x_{1}, \ldots, x_{k}\right)$ near $f(\psi)=g(\psi)$. We know that

$$
\theta_{\psi}\left(x_{i} \circ f\right)=\left(\theta_{\psi} \circ f^{*}\right)\left(x_{i}\right)=\left(\theta_{\psi} \circ g^{*}\right)\left(x_{i}\right)=\theta_{\psi}\left(x_{i} \circ g\right)
$$

for $i=1, \ldots, k$. Therefore, $x_{i} \circ f-x_{i} \circ g \in \mathscr{N}_{\psi}$. But then $x_{i} \circ f-x_{i} \circ g \in$ $\mathscr{N}_{\gamma}$ for all $\gamma \in X(A) \cap U_{i}$, where $U_{i}$ is an open neighborhood of $\psi$, for $i=1, \ldots, k$. If $U=\bigcap_{i=1}^{k} U_{i}$ then $f(\gamma)=g(\gamma)$ when $\gamma \in U \cap X(A)$ and also $\theta_{\gamma} \circ f^{*}-\theta_{\gamma} \circ g^{*}: \mathscr{O}_{f(\gamma)} \rightarrow A_{\gamma}$ is null because it is zero over the set of generators $x_{1}, \ldots, x_{k}$ :

$$
\left(\theta_{\gamma} \circ f^{*}-\theta_{\gamma} \circ g^{*}\right)\left(x_{i}\right)=\theta_{\gamma}\left(x_{i} \circ f-x_{i} \circ g\right)=0 .
$$

We may suppose $U$ contained in $W \cap V$. Then

$$
u \in s_{f}(U)=s_{g}(U) \subset s_{f}(W) \cap s_{g}(V) .
$$

4.2. Definition. The sheaf $\mathscr{A}_{M}$ is the sheaf over $X(A)$ whose total space and projection are as above, and whose topology has the basis

$$
\left\{s_{f}(W): W \text { is open and } f \in \mathscr{O}(W, M)\right\} .
$$

4.3. Proposition. Let $M$ and $N$ be complex analytic manifolds, and $h: M \rightarrow N$ an analytic map. Then $h$ induces a sheaf morphism

$$
\mathscr{A}_{h}: \mathscr{A}_{M} \rightarrow \mathscr{A}_{N}
$$

Also, $\mathscr{A}_{d \circ h}=\mathscr{A}_{d} \circ \mathscr{A}_{h}$ and $\mathscr{A}_{\text {id }}=$ identity of $\mathscr{A}_{M}$. 
Proof. Define

$$
\mathscr{A}_{h}(u)=h_{\psi}(u), \quad \text { if } \pi(u)=\psi .
$$

Obviously $\mathscr{A}_{h}$ commutes with the projections. Let's see the continuity of $\mathscr{A}_{h}$. Let $u \in \mathscr{A}_{M}$ and $v=\mathscr{A}_{h}(u)$. To fix notation, say $u=\alpha_{\psi}(f)$ and $v=h_{\psi}\left(\alpha_{\psi}(f)\right)=\beta_{\psi}(h \circ f)$.

Let $s_{g}(V)$ be a neighborhood of $v$, that is, $V$ is an open neighborhood of $\psi$, and $g \in \mathscr{O}_{\psi}^{N}$ is such that $\beta_{\psi}(g)=v$. Since

$$
s_{g}(\psi)=\beta_{\psi}(g)=v=\beta_{\psi}(g \circ f)=s_{h \circ f}(\psi)
$$

we know there is a neighborhood $U$ of $\psi$ over which $s_{g}$ and $s_{h \circ f}$ coincide. We may also suppose that $U$ is contained in $V$. Then $s_{f}(U)$ is a neighborhood of $u$, and $\mathscr{A}_{h}\left(s_{f}(U)\right) \subset s_{g}(V)$, for if $\gamma \in U$,

$$
\mathscr{A}_{h}\left(s_{f}(\gamma)\right)=s_{h \circ f}(\gamma)=s_{g}(\gamma) .
$$

$\mathscr{A}_{d \circ h}=\mathscr{A}_{d} \circ \mathscr{A}_{h}$ and $\mathscr{A}_{\text {id }}=$ identity of $\mathscr{A}_{M}$ are simple.

5. The spectral set $A_{M}$. We have, for any complex analytic manifold $M$, and any commutative Banach algebra $A$ the following definition of the spectral set $A_{M}$.

5.1. Definition. $A_{M}=H^{0}\left(X(A), \mathscr{A}_{M}\right)$.

5.2. Proposition. Let $M$ and $N$ be complex analytic manifolds, and $h: M \rightarrow N$ an analytic map. Then $h$ induces a map

$$
A_{h}: A_{M} \rightarrow A_{N}
$$

and we also have $A_{d \circ h}=A_{d} \circ A_{h}$ and $A_{\mathrm{id}}=$ identity of $A_{M}$.

Proof. The proof is trivial, given the continuity of $\mathscr{A}_{h}$. Simply define

$$
A_{h}(s)=\mathscr{A}_{h} \circ s \text {. }
$$

The functoriality of the construction $M \mapsto A_{M}$ is clear.

The following facts, which will be needed later on, are also easy to prove.

5.3. Proposition. (i) If $S$ is a submanifold of $M$, then $A_{S}$ is contained in $A_{M}$.

(ii) $A_{M \times N}$ may be identified with $A_{M} \times A_{N}$.

(iii) $M$ is contained in $A_{M}$. 
(iv) The "functional calculus" map

$$
\theta_{M}: \mathscr{O}(X(A), M) \rightarrow A_{M}
$$

defined by $f \mapsto s_{f}$, is surjective.

We shall compare the spectral set $A_{M}$, when $M$ is a submanifold of $\mathrm{C}^{n}$, to those defined in [8]:

$$
A^{M}=\left\{a \in A^{n}: \operatorname{sp}(a) \subset M\right\}
$$

and in [10]:

$$
A_{M}^{T}=\left\{a \in A^{n}: a=\theta^{n}(f) \text {, for some } f \in \mathscr{O}(X(A), M)\right\} .
$$

It is well known $[6,10]$ that $A^{M}=A_{M}^{T}+\operatorname{Rad}(A)^{n}$, so both coincide if $A$ is semisimple. They also coincide if $M$ is an open subset of $\mathbf{C}^{n}$.

First we shall take a closer look at spectral sets $A_{\Omega}$, where $\Omega$ is an open subset of $\mathbf{C}^{n}$. Let $z=\left(z_{1}, \ldots, z_{n}\right): \Omega \rightarrow \mathbf{C}^{n}$ be the inclusion map. Then

$$
\begin{aligned}
& \theta_{\psi}^{n}(f)=\theta_{\psi}^{n}(z \circ f)=\left(\left(\theta_{\psi} \circ f^{*}\right)\left(z_{1}\right), \ldots,\left(\theta_{\psi} \circ f^{*}\right)\left(z_{n}\right)\right) \quad \text { and } \\
& \theta_{\psi}^{n}(g)=\theta_{\psi}^{n}(z \circ g)=\left(\left(\theta_{\psi} \circ g^{*}\right)\left(z_{1}\right), \ldots,\left(\theta_{\psi} \circ g^{*}\right)\left(z_{n}\right)\right)
\end{aligned}
$$

so $\theta_{\psi}^{n}(f)=\theta_{\psi}^{n}(g)$ if and only if $\alpha_{\psi}(f)=\alpha_{\psi}(g)$. Looking at the diagram

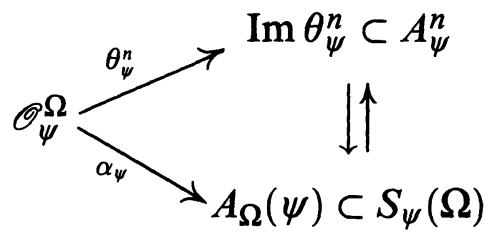

we see that $A_{\Omega}(\psi)$ may be identified with the image of the map

$$
\theta_{\psi}^{n}: \mathscr{O}_{\psi}^{\Omega} \rightarrow A_{\psi}^{n}
$$

Now our basic construction gives

5.4. Proposition. $A_{\mathrm{C}}=A$.

Proof. We have the sheaf map $\mathscr{O} \rightarrow \mathscr{A}_{\mathbf{C}}$ given by $f \mapsto s_{f}$ on each open subset of $X(A)$, but since $s_{f}(\psi)=\alpha_{\psi}(f)=\left(f(\psi), \theta_{\psi} \circ f^{*}\right)$ for each $\psi$ in the domain of $f$, we can identify the stalks $A_{\mathbf{C}}(\psi)$ and $A_{\psi}$ 
by the rule $s_{f}(\psi) \mapsto \theta_{\psi}(f)$. Hence sheaves $\mathscr{A}$ and $\mathscr{A}_{\mathbf{C}}$ are isomorphic. From now on we will write simply $\mathscr{A}$.

5.5. Corollary. $A_{\mathbf{C}^{n}}=A^{n}$.

5.6. Proposition. Let $\Omega$ be an open subset of $\mathbf{C}^{n}$. Then $A_{\Omega}=A^{\Omega}=$ $A_{\Omega}^{T}$

Proof. We know that $A_{\Omega}$ is contained in $A^{n}$. Also, the second equality holds, so we need only prove

$$
A_{\Omega}^{T} \subset A_{\Omega} \subset A^{\Omega} .
$$

If $a=\theta^{n}(f)$, for some $f \in \mathscr{O}(X(A), \Omega), a$ is identified with the section $s \in A_{\Omega}$ given by $s(\psi)=\theta_{\psi}^{n}(f) \in A_{\Omega}(\psi)$.

Now let $s \in A_{\Omega}$ be identified with $a=\left(a_{1}, \ldots, a_{n}\right) \in A^{n}$, and take $\psi \in X(A) . s(\psi) \in A_{\Omega}(\psi)=\operatorname{Im} \theta_{\psi}^{n}$; say $s(\psi)=\theta_{\psi}^{n}(f)$. Then it may be seen that

$$
\left(\psi\left(a_{1}\right), \ldots, \psi\left(a_{n}\right)\right)=f(\psi)
$$

which belongs to $\Omega$, for $f \in \mathscr{O}_{\psi}^{\Omega}$. Therefore $\operatorname{sp}(a) \subset \Omega$.

Note that we obtain, for example, $A_{\mathrm{GL}_{n}(\mathbf{C})}=\mathrm{GL}_{n}(A)$ (invertible $n \times n A$-valued matrices), and $A_{\mathbf{C}^{*}}=A^{-1}$ (the invertible elements of $A)$.

We can now prove that our definition generalizes Taylor's. Observe first that the previous identifications give the commutative triangle

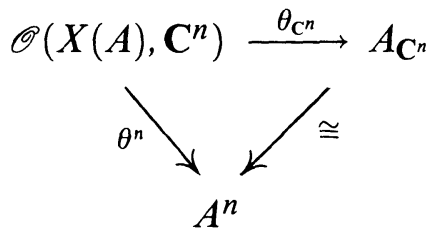

where the isomorphism is simply $s_{f} \mapsto \theta^{n}(f)$.

5.7. Proposition. If $M$ is a closed submanifold of $\mathbf{C}^{n}$, then $A_{M}=$ $A_{M}^{T}$ under the previous identification. 
Proof. The proof can be obtained by inspection of the commutative diagram

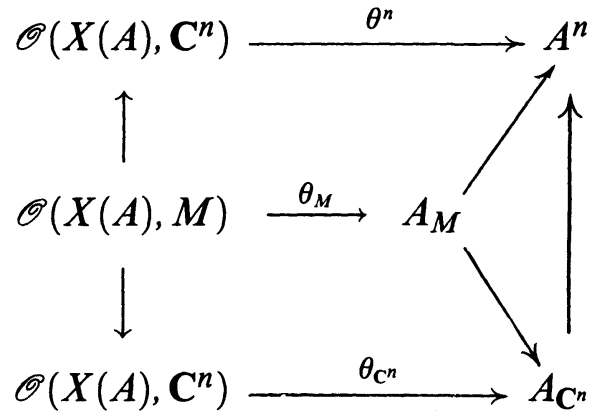

A notion of spectrum for elements of $A_{M}$, may be defined as follows.

5.8. Definition For every $\psi \in X(A)$, set

$$
\psi^{M}: A_{M} \rightarrow M
$$

such that $\psi^{M}(a)=f(\psi)$, if $a(\psi)=\alpha_{\psi}(f)$. Define the spectrum of an element of $A_{M}$ as the subset of $M$ :

$$
\operatorname{sp}^{M}(a)=\left\{\psi^{M}(a): \psi \in X(A)\right\} .
$$

Note that when $M=\mathbf{C}, \psi^{M}=\psi$ and if $M$ is a subset of $\mathbf{C}^{n}$, and $a \in A_{M}$ is identified with an element of $A^{n}$, then $\operatorname{sp}^{M}(a)$ is the joint spectrum of this $n$-tuple.

Note also that if $h \in \mathcal{O}\left(\operatorname{sp}^{M}(a), N\right)$ we may define $h(a)$ as the element of $A_{N}$ given by $h(a)(\psi)=\beta_{\psi}(h \circ f)$, if $a=\alpha_{\psi}(f)$. This is a well-defined functional calculus.

EXAMPLE. If $\mathbf{P}_{1}$ denotes the complex projective line, the elements of $A$ may be identified with those $a \in A_{\mathbf{P}_{1}}$ such that $\infty \notin \operatorname{sp}^{\mathbf{P}_{1}}(a)$. Any meromorphic function $h$ may be evaluated at $a$; of course $h(a)$ will not be an element of $A$ unless $\infty \notin \operatorname{sp}^{\mathbf{P}_{1}}(h(a))=h(\operatorname{sp}(a))$. For instance, the function

$$
1 / z: \mathbf{P}_{1} \rightarrow \mathbf{P}_{1}
$$

induces

$$
1 / a: A_{\mathbf{P}_{1}} \rightarrow A_{\mathbf{P}_{1}} \text {. }
$$

When $0 \notin \operatorname{sp}(x), 1 / x$ is an element of $A$ and coincides with $x^{-1}$.

We note that $A_{\mathbf{P}_{1}}$ is larger than Glickfeld's $A_{\infty}$ [5]. The reason for this will become apparent in the next section.

Define a generalized Gelfand transform as follows.

$$
\Gamma_{M}: A_{M} \rightarrow \mathscr{C}(X(A), M) / \Gamma_{M}(a)(\psi)=\psi^{M}(a) .
$$


Note that if $a(\psi)=\alpha_{\psi}(f)$, then $\Gamma_{M}(a)(\gamma)=f(\gamma)$ for characters $\gamma$ which are sufficiently close to $\psi$. Hence, $\Gamma_{M}(a)$ is continuous.

To conclude this section, we introduce a topology on $A_{M}$ for semisimple $A$. Suppose $a \in A_{M}$, and that $U$ is an open neighborhood of the diagonal $\Delta \subset M \times M$. Then define

$$
U_{a}=\left\{b \in A_{M}:(a, b) \in A_{U}\right\} \text {. }
$$

If we take $\mathscr{E}$ to be the set of all open neighborhoods of $\Delta$, then

$$
\mathscr{E}_{a}=\left\{U_{a}: U \in \mathscr{E}\right\}
$$

is a basis for neighborhoods of $a$.

With this topology on $A_{M}$ it is easy to prove that both the generalized Gelfand transform $\Gamma_{M}$, and the functional calculus $\theta_{M}$, are continuous. Also, $A_{\mathbf{C}}=A$ topologically (for semisimple $A$ ). We consider $\mathscr{C}(X(A), M)$ with the compact-open topology, and $\mathscr{O}(X(A), M)$ with the direct limit topology arising when every function is considered finitely determined (that is, a function of a finite number of variables) [7].

If $F$ is a complex homogeneous space, even though not a submanifold of $\mathbf{C}^{n}$, we obtain the Novodvorskii-Taylor theorem for the spectral set $A_{F}$.

5.9. Theorem. Let $A$ be semisimple and let $F$ be a complex homogeneous space. Let $\left[A_{F}\right]$ denote the set of connectivity components of $A_{F}$ and $[X(A), F]$ the set of homotopy classes of maps from $X(A)$ to $F$. Then there is a bijection

$$
\left[A_{F}\right] \rightarrow[X(A), F]
$$

Proof. The map $\left[A_{F}\right] \rightarrow[X(A), F]$ is induced by the generalized Gelfand transform $\Gamma_{F}$. Bijectivity follows directly from Proposition 2.6 of [10].

6. Homogeneous spectral sets. In this section we take a closer look at spectral sets $A_{F}$, where $F$ is a homogeneous space; that is, a quotient of an analytic Lie group by a (not necessarily normal) closed Lie subgroup. We also consider spectral sets obtained from principal fiber bundles.

First, suppose $G$ is an analytic Lie group. Then the analytic maps

$$
\begin{aligned}
& p: G \times G \rightarrow G \quad \text { (product) and } \\
& i: G \rightarrow G \quad \text { (inverse) }
\end{aligned}
$$


induce the corresponding maps

$$
A_{G} \times A_{G} \rightarrow A_{G} \text { and } A_{G} \rightarrow A_{G} .
$$

That $A_{G}$, with these operations, is a group, is easily verified by applying the functor $A_{(\cdot)}$ to suitable commutative diagrams.

If $H$ is another Lie group and $h: H \rightarrow G$ is a Lie group morphism, then $A_{h}: A_{H} \rightarrow A_{G}$ is a group morphism. When $H$ is a Lie subgroup of $G, A_{H}$ is a subgroup of $A_{G}$.

It is natural to ask, in this context, if the "exact sequence"

$$
1 \rightarrow H \rightarrow G \rightarrow G / H \rightarrow 1
$$

induces an exact sequence

$$
1 \rightarrow A_{H} \rightarrow A_{G} \rightarrow A_{G / H} \rightarrow 1 .
$$

In other words, can the homogenous spectral set $A_{G / H}$ be described as $A_{G} / A_{H}$ ? The answer in general is no. An idea of why this is so may be obtained by trying to prove that

$$
\mathscr{O}(X(A), G) \rightarrow \mathscr{O}(X(A), G / H)
$$

is surjective. It is not, and the main setback is that $X(A)$ can be too large.

We can, however, prove exactness of (1) in the local case, and this will lead us to see just how inexact sequence (1) is. Actually, we shall prove a stronger form of this result, valid for principal fiber bundles.

Let $\pi: P \rightarrow M$ be an analytic principal fiber bundle with group $H$ acting on $P$. Fix $z \in P$, and let $i: H \rightarrow P$ be the resulting map $i(c)=c z$. We then have the exact sequence

$$
1 \rightarrow H \stackrel{i}{\rightarrow} P \stackrel{\pi}{\rightarrow} M \rightarrow 1 .
$$

The resulting sequence of spectral sets is locally exact; that is, we obtain the following lemma.

6.1. LEMMA. Let $P \rightarrow M$ be an analytic principal fiber bundle with group $H$, and let $\psi \in X(A)$. Then, with notation as above, the following sequence is exact.

$$
1 \rightarrow A_{H}(\psi) \stackrel{i_{\psi}}{\rightarrow} A_{P}(\psi) \stackrel{\pi_{\psi}}{\rightarrow} A_{M}(\psi) \rightarrow 1 .
$$

Proof. To fix notation, say $A_{H}(\psi)=\operatorname{Im} \alpha_{\psi}, A_{P}(\psi)=\operatorname{Im} \beta_{\psi}$, and $A_{M}(\psi)=\operatorname{Im} \gamma_{\psi}$. Note that the group $A_{H}(\psi)$ acts on $A_{P}(\psi), \alpha_{\psi}$ is a group morphism, and

$$
\beta_{\psi}(h f)=\alpha_{\psi}(h) \beta_{\psi}(f), \quad \text { for all } h \in \mathscr{O}_{\psi}^{H}, f \in \mathscr{O}_{\psi}^{P} .
$$


We shall start with the injectivity of $i_{\psi}$. Suppose $i_{\psi}\left(\alpha_{\psi}(h)\right)=$ $i_{\psi}\left(\alpha_{\psi}(g)\right)$, that is,

$$
\begin{aligned}
\left(i(h(\psi)), \theta_{\psi} \circ(i \circ h)^{*}\right) & =\beta_{\psi}(i \circ h)=\beta_{\psi}(i \circ g) \\
& =\left(i(g(\psi)), \theta_{\psi} \circ(i \circ g)^{*}\right) .
\end{aligned}
$$

Then $h(\psi)=g(\psi)=c$, for $i$ is injective, and

$$
\theta_{\psi} \circ h^{*} \circ i^{*}=\theta_{\psi} \circ g^{*} \circ i^{*}
$$

as morphisms from $\mathscr{O}_{c z}$ to $A_{\psi}$. But $i^{*}: \mathscr{O}_{c z} \rightarrow \mathscr{O}_{c}$ is an epimorphism, so $\theta_{\psi} \circ h^{*}=\theta_{\psi} \circ g^{*}$. We then have

$$
\alpha_{\psi}(h)=\left(h(\psi), \theta_{\psi} \circ h^{*}\right)=\left(g(\psi), \theta_{\psi} \circ g^{*}\right)=\alpha_{\psi}(g) .
$$

To see the surjectivity of $\pi_{\psi}$, let $\gamma_{\psi}(l) \in A_{M}(\psi)$ and suppose $\sigma$ is a local section of $\pi$ near $l(\psi)$. Let $f=\sigma \circ l \in \mathscr{O}_{\psi}^{P}$. Then

$$
\begin{aligned}
\pi_{\psi}\left(\beta_{\psi}(f)\right) & =\pi_{\psi}\left(\beta_{\psi}(\sigma \circ l)\right)=\pi_{\psi}\left(\sigma_{\psi}\left(\gamma_{\psi}(l)\right)\right) \\
& =(\pi \circ \sigma)_{\psi}\left(\gamma_{\psi}(l)\right)=\gamma_{\psi}(l) .
\end{aligned}
$$

For the exactness in $A_{P}(\psi)$, note first that $\pi_{\psi} \circ i_{\psi}=(\pi \circ i)_{\psi}=$ the constant $\pi(z)$. We must now prove that given $f$ and $g$ in $\mathscr{O}_{\psi}^{P}$ such that $\pi_{\psi}\left(\beta_{\psi}(f)\right)=\pi_{\psi}\left(\beta_{\psi}(g)\right)$, there exits an $h \in \mathscr{O}_{\psi}^{H}$ with $\beta_{\psi}(g)=$ $\alpha_{\psi}(h) \beta_{\psi}(f)$. We have

$$
\gamma_{\psi}(\pi \circ f)=\pi_{\psi}\left(\beta_{\psi}(f)\right)=\pi_{\psi}\left(\beta_{\psi}(g)\right)=\gamma_{\psi}(\pi \circ g)
$$

so $\pi(f(\psi))=\pi(g(\psi))$. Let $V$ be an open neighborhood of this point, and

$$
\sigma: V \rightarrow P, \quad \text { a section of } \pi
$$

$\gamma_{\psi}(\pi \circ f)=\gamma_{\psi}(\pi \circ g)$ belongs to $A_{V}(\psi)$. Applying $\sigma_{\psi}$ we obtain

$$
\beta_{\psi}(\sigma \circ \pi \circ f)=\beta_{\psi}(\sigma \circ \pi \circ g) \text {. }
$$

Now for each $\gamma$ in a neighborhood of $\psi, \pi(\sigma(\pi(f(\gamma))))=\pi(f(\gamma))$; therefore for every such $\gamma$ there is a unique $h_{1}(\gamma) \in H$ such that

$$
\sigma(\pi(f(\gamma)))=h_{1}(\gamma) f(\gamma) .
$$

Since $P$ is locally $H \times M$, the mapping $\gamma \mapsto h_{1}(\gamma)$ may be seen to be analytic, so $h_{1} \in \mathscr{O}_{\psi}^{H}$. Likewise,

$$
\sigma(\pi(g(\gamma)))=h_{2}(\gamma) g(\gamma)
$$

with $h_{2} \in \mathscr{O}_{\psi}^{H}$, and we have

$$
\alpha_{\psi}\left(h_{2}\right) \beta_{\psi}(g)=\beta_{\psi}(\sigma \circ \pi \circ g)=\beta_{\psi}(\sigma \circ \pi \circ f)=\alpha_{\psi}\left(h_{1}\right) \beta_{\psi}(f) .
$$


Now take $h=h_{2}^{-1} h_{1} \in \mathscr{O}_{\psi}^{H}$, and obtain

$$
\begin{aligned}
\alpha_{\psi}(h) \beta_{\psi}(f) & =\alpha_{\psi}\left(h_{2}^{-1}\right) \alpha_{\psi}\left(h_{1}\right) \beta_{\psi}(f) \\
& =\alpha_{\psi}\left(h_{2}^{-1}\right) \alpha_{\psi}\left(h_{2}\right) \beta_{\psi}(g)=\beta_{\psi}(g) .
\end{aligned}
$$

This concludes the proof.

The following corollary is now trivial.

6.2. Corollary. Let $G$ be a Lie group, and $H$ a closed Lie subgroup. The exact sequence

$$
1 \rightarrow H \rightarrow G \rightarrow G / H \rightarrow 1
$$

induces an exact sequence of local spectral sets

$$
1 \rightarrow A_{H}(\psi) \rightarrow A_{G}(\psi) \rightarrow A_{G / H}(\psi) \rightarrow 1
$$

for any $\psi \in X(A)$.

We now obtain our main theorem, which shows the inexactness of (1).

6.3. THEOREM. (i) Let $P \rightarrow M$ be an analytic principal fiber bundle with group $H$ acting on $P$. Then the following sequence is exact

$$
1 \rightarrow A_{H} \rightarrow A_{P} \rightarrow A_{M} \rightarrow H^{1}\left(X(A), \mathscr{A}_{H}\right)
$$

(ii) Let $G$ be a Lie group, and $H$ a closed Lie subgroup. Then the following sequence is exact

$$
1 \rightarrow A_{H} \rightarrow A_{G} \rightarrow A_{G / H} \rightarrow H^{1}\left(X(A), \mathscr{A}_{H}\right) \rightarrow H^{1}\left(X(A), \mathscr{A}_{G}\right) .
$$

Proof. Applying the lemma and corollary, obtain the exact sequences of sheaves

$$
1 \rightarrow \mathscr{A}_{H} \rightarrow \mathscr{A}_{P} \rightarrow \mathscr{A}_{M} \rightarrow 1
$$

and

$$
1 \rightarrow \mathscr{A}_{H} \rightarrow \mathscr{A}_{G} \rightarrow \mathscr{A}_{G / H} \rightarrow 1 .
$$

Now sheaf cohomology theory gives (i) and (ii). See [4].

7. Examples and applications. In some applications of the preceding theorems, sheaves and spectral sets appear where the manifold is 
simply a discrete set $D$. It will be convenient, therefore, to start by considering such sheaves and spectral sets.

Note that the germ of an analytic function taking values in a discrete set $D$ is simply a germ of a constant, so the set $\mathscr{O}_{\psi}^{D}$, and the map

$$
\alpha_{\psi}: D \rightarrow S_{\psi}(D)
$$

is $d \mapsto\left(d, \theta_{\psi} \circ e_{d}\right)$. Hence, $A_{D}(\psi)$ may be identified with $D$. The sheaf $\mathscr{A}_{D}$ is then simply the sheaf $D$ over $X(A)$, and

$$
A_{D}=D^{\pi_{0}(X(A))} \text {. }
$$

Since in the applications of the preceding section, there appear cohomology groups of the not very manageable sheaves $\mathscr{A}_{G}$, we note that $H^{1}\left(X(A), \mathscr{A}_{G}\right)=H^{1}\left(X(A), \mathscr{O}^{G}\right)=H^{1}\left(X(A), \mathscr{C}^{G}\right)$. To prove this, use [4] to see that for any Stein space $S$ contained in $\mathbf{C}^{k}, H^{1}\left(S, \mathscr{O}^{G}\right)=$ $H^{1}\left(S, \mathscr{C}^{G}\right)$, then a direct limit argument such as the one used in $\S 2$ gives $H^{1}\left(X(A), \mathscr{O}^{G}\right)=H^{1}\left(X(A), \mathscr{C}^{G}\right)$, and finally note that the following diagram is commutative

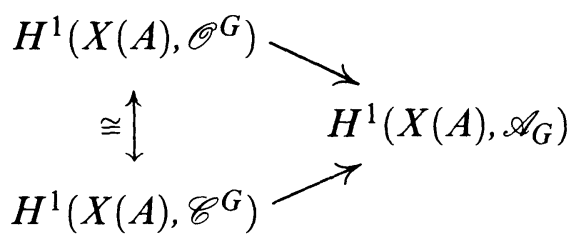

We are now ready to present a few applications of our results.

EXAMPLE 1. The Arens-Royden theorem.

The exact sequence

$$
0 \rightarrow \mathbf{Z} \rightarrow \mathbf{C} \stackrel{\exp }{\longrightarrow} \mathbf{C}^{*} \rightarrow 0
$$

induces

$$
0 \rightarrow A_{\mathbf{Z}} \rightarrow A_{\mathbf{C}} \stackrel{\exp }{\longrightarrow} A_{\mathbf{C}^{*}} \rightarrow H^{1}\left(X(A), \mathscr{A}_{\mathbf{Z}}\right) \rightarrow H^{1}(X(A), \mathscr{A}) \rightarrow \cdots,
$$

which may also be written as

$$
0 \rightarrow \mathbf{Z}^{\pi_{0}(X(A))} \rightarrow A \stackrel{\exp }{\rightarrow} A^{-1} \stackrel{d}{\rightarrow} H^{1}(X(A), \mathbf{Z}) \rightarrow 0 \rightarrow \cdots .
$$

Therefore, $H^{1}(X(A), \mathbf{Z})=A^{-1} / \operatorname{Ker} d=A^{-1} / \exp (A)$.

Continuing the sequence we also obtain

$$
H^{p}\left(X(A), \mathscr{A}_{\mathbf{C}^{*}}\right)=H^{p+1}(X(A), \mathbf{Z}), \quad \text { for all } p \geq 1 .
$$

EXAMPLE 2. $n$-dimensional projective space. 
$\left(\mathbf{C}^{n+1}\right)^{*} \rightarrow \mathbf{P}_{n}$ is a principal fiber bundle with group $\mathbf{C}^{*}$ acting on $\left(\mathbf{C}^{n+1}\right)^{*}$. Then the following sequence

$$
1 \rightarrow A_{\mathbf{C}^{*}} \rightarrow A_{\left(\mathbf{C}^{n+1}\right)^{*}} \rightarrow A_{\mathbf{P}_{n}} \rightarrow H^{1}\left(X(A), \mathscr{A}_{\mathbf{C}^{*}}\right),
$$

which may also be written as

$$
1 \rightarrow A^{-1} \rightarrow \operatorname{Uni}\left(A^{n+1}\right) \rightarrow A_{\mathbf{P}_{n}} \stackrel{c}{\rightarrow} H^{2}(X(A), \mathbf{Z})=\operatorname{Pic}(A)
$$

is exact. Here $\operatorname{Uni}\left(A^{n+1}\right)=\left\{\left(a_{1}, \ldots, a_{n+1}\right) \in A^{n+1}: \sum_{i=1}^{n+1} b_{i} a_{i}=1\right.$, for some $\left.b_{1}, \ldots, b_{n+1} \in A\right\}$. The elements $x \in A_{\mathbf{P}_{n}}$ may therefore be considered one-dimensional fibered spaces over $X(A)$, and $c(x)$ can be thought of as the Chern class of $x$.

Note that for $n=1$, we obtain: the homogeneous spectral set $A_{\mathbf{P}_{1}}$ is, in general, larger than the Riemann sphere $A_{\infty}=\operatorname{Uni}\left(A^{2}\right) / A^{-1}$ defined by Glickfeld [5]. $A_{\infty}$ and $A_{\mathbf{P}_{1}}$ coincide if and only if $H^{2}(X(A), \mathbf{Z})=0$.

EXAMPLE 3. $n$th roots of invertible elements.

The exact sequence

$$
1 \rightarrow G_{n} \rightarrow \mathbf{C}^{*} \stackrel{z \mapsto z^{n}}{\longrightarrow} \mathbf{C}^{*} \rightarrow 1
$$

gives rise to

$$
1 \rightarrow G_{n}^{\pi_{0}(X(A))} \rightarrow A^{-1} \stackrel{p}{\rightarrow} A^{-1} \rightarrow H^{1}\left(X(A), G_{n}\right) \rightarrow \cdots
$$

where $p(a)=a^{n}$. Therefore, for all invertible elements of $A$ to have $n$th roots, it is necessary and sufficient that $H^{1}\left(X(A), G_{n}\right)=0$.

EXAMPLE 4. Interior automorphism of $\mathrm{GL}_{n+1}(A)$.

The group of automorphisms of $\mathrm{GL}_{n+1}(\mathrm{C})$ may be regarded as the projective general linear group $\mathrm{PGL}_{n}(\mathbf{C})$ given by the action of $\mathrm{C}^{*}$ on $\mathrm{GL}_{n+1}(\mathbf{C})$,

$$
1 \rightarrow \mathrm{C}^{*} \rightarrow \mathrm{GL}_{n+1}(\mathrm{C}) \stackrel{\pi}{\rightarrow} \mathrm{PGL}_{n}(\mathrm{C}) \rightarrow 1 \text {. }
$$

The surjectivity of $\pi$ says that all such automorphisms are interior. The exact sequence induces

$$
1 \rightarrow A^{-1} \rightarrow \mathrm{GL}_{n+1}(A) \rightarrow A_{\mathrm{PGL}_{n}(\mathrm{C})} \rightarrow H^{2}(X(A), \mathbf{Z})=\operatorname{Pic}(A)
$$

which is comparable to the analogous exact sequence in [2].

\section{REFERENCES}

[1] G. R. Allan, A form of local characterization of Gelfand transforms, J. London Math. Soc., 43 (1968), 623-625.

[2] H. Bass, Lectures on topics in algebraic K-theory, Tata Institute, Bombay, 1967. 
[3] I. G. Craw, A condition equivalent to the continuity of characters on a Frechet algebra, Proc. London Math. Soc., (3) 22 (1971), 425-464.

[4] O. Forster and K. Ramspott, Okasche Paare von Garben nicht-abelscher Gruppen, Inventiones Math., 1 (1966), 260-286.

[5] B. W. Glickfeld, The Riemann sphere of a commutative Banach algebra, Trans. Amer. Math. Soc., 134 (1968), 1-28.

[6] A. Larotonda and I. Zalduendo, Spectral sets as Banach manifolds, Pacific J. Math., 120, No. 2 (1985), 401-416.

[7] , The holomorphic functional calculus, Rev. de la Un. Mat. Arg., 31 (1984), 139-148.

[8] E. Novodvorskii, Certain homotopical invariants of spaces of maximal ideals, Mat. Z., 1 (1967), 487-494.

[9] I. Raeburn, The relationship between a commutative Banach algebra and its maximal ideal space, J. Funct. Analysis, 25 (1977), 366-390.

[10] J. L. Taylor, Topological invariants of the maximal ideal space of a Banach algebra, Adv. in Math., 19 (1976), 149-206.

[11] L. Waelbroeck, Topological vector spaces and algebras, Lecture Notes in Mathematics No. 230, Springer, New York, 1971.

Received March 6, 1987.

Universidad DE BUENos Aires

(1428) Capital Federal, Argentina

Current address of the second author: UCLA

Los Angeles, CA 90024

USA 


\section{PACIFIC JOURNAL OF MATHEMATICS EDITORS}

V. S. VARADARAJAN (Managing Editor) University of California Los Angeles, CA 90024

HeRbert Clemens

University of Utah

Salt Lake City, UT 84112

R. FINN

Stanford University

Stanford, CA 94305
ROBION KIRBY

University of California

Berkeley, CA 94720

C. C. MOORE

University of California

Berkeley, CA 94720

HAROLD STARK

University of California, San Diego

La Jolla, CA 92093

\section{ASSOCIATE EDITORS}
R. ARENS
E. F. BECKENBACH
B. H. NEUMANN
F. Wolf
K. YOSHIDA (1906-1982)

\section{SUPPORTING INSTITUTIONS}

UNIVERSITY OF ARIZONA UNIVERSITY OF OREGON

UNIVERSITY OF BRITISH COLUMBIA UNIVERSITY OF SOUTHERN CALIFORNIA

CALIFORNIA INSTITUTE OF TECHNOLOGY UNIVERSITY OF CALIFORNIA MONTANA STATE UNIVERSITY STANFORD UNIVERSITY

UNIVERSITY OF NEVADA, RENO UNIVERSITY OF HAWAII NEW MEXICO STATE UNIVERSITY UNIVERSITY OF TOKYO UNIVERSITY OF UTAH OREGON STATE UNIVERSITY WASHINGTON STATE UNIVERSITY UNIVERSITY OF WASHINGTON 


\section{Pacific Journal of Mathematics}

Vol. 134, No. $1 \quad$ May, 1988

Marco Abate, Annular bundles . ............................

Ralph Cohen, Wen Hsiung Lin and Mark Mahowald, The Adams

spectral sequence of the real projective spaces

Harry Joseph D'Souza, Threefolds whose hyperplane sections are elliptic surfaces .........................................57

Theodore Gerard Faticoni, Localization in finite-dimensional FPF rings . . . 79

Daniel Hitt, Invariant subspaces of $\mathscr{H}^{2}$ of an annulus ................ 101

Ellen Kirkman and James J. Kuzmanovich, On the global dimension of fibre products

Angel Rafael Larotonda and Ignacio Zalduendo, Homogeneous spectral sets and local-global methods in Banach algebras

Halsey Lawrence Royden, Jr., Invariant subspaces of $\mathscr{H}^{p}$ for multiply

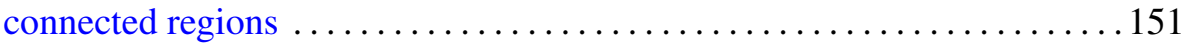

Jane Sangwine-Yager, A Bonnesen-style inradius inequality in 3-space . . . . 173

Stefano Trapani, Holomorphically convex compact sets and cohomology

Thomas Vogel, Uniqueness for certain surfaces of prescribed mean

curvature 